\title{
ETUDE ETHNOGRAPHIQUE DE L'ITOMBWE (DISTRICT DU KIVU, CONGO BELGE)
}

\author{
Pat Frédéric HautmanN
}

Avec 2 figures

Une des régions les moins touchées par la civilisation que nous avons pu étudier au cours d'un long séjour en Afrique centrale est le massif montagneux au nord-ouest du lac Tanganyika, le pays appelé l' «Itombwe». C'est la région des sources de deux grands affluents du Congo, l'Úlindi et l'Elila. Ce haut plateau est limité à l'est par la vallée de la Ruzizi et la partie septentrionale du lac Tanganyika, à l'ouest par la grande forêt du Maniema.

La carte ethnographique, éditée en 1939 par le "Comité national du Kivu», ne donne que des renseignements imprécis au sujet de ce pays. En fait, il est habité par plusieurs peuplades: d'une part par les Bafulero dont on distingue ceux de la plaine de la Ruzizi et ceux de la montagne, deux groupes bien distincts l'un de l'autre. D'autre part, le centre montagneux de ce massif, couvert de pâturages verdoyantes pendant toute l'année, a attiré les peuplades pasteurs du Ruanda-Urundi, les Banya-R uanda et les Barundi, avec leurs troupeaux de bétail Watussi. La pente du sud-est est habitée par les Babembe, apparentés aux Warega de l'ouest, grands chasseurs et anciens anthropophages. En symbiose avec les Bafulero de montagne habitent les Bambuti, derniers restes de la population autochthone des pygmées, qui sont très métissés et peu distincts de leurs maîtres.

D'où qu'on vienne, on n'y entre qu'à pied, en caravane, "en safari». En venant du Kivu du Nord, on quitte sa voiture soit à Uvira, soit à Sanghe, tous deux villages situés au pied des chaînes de montagnes qui s'élèvent dans la partie occidentale de la vallée de la Ruzizi, rivière qui, par la formation du "Graben", a été détournée de son écoulement antérieur vers le Nord.

Pour monter au massif de l'Itombwe, peu accessible, nous avons choisi la montée la plus facile de Sanghe que les pâtres du Ruanda-Urundi préfèrent. On met 5 à 6 jours de marche jusqu'au cœur de ce pays, avec sa petite rivière "Luemba». L'exploitation d'une mine d'or depuis 1938 à cet endroit n'a guère apporté des modifications à la structure si multiforme de cette population.

$\mathrm{Au}$ premier jour, le safari passe par plusieurs villages Bafulero. Plus on monte, plus la population change d'aspect. On ne voit plus les malheureux trypanosés de la plaine indigènes atteints de la maladie du sommeil - car la glossina palpalis n'existe pas sur les hauteurs; elle se limite aux bords de la plaine de la Ruzizi. En même temps disparaît un autre fléau, l'alcoolisme: le bananier du Kivu, cultivé dans les régions inférieures à $1800 \mathrm{~m}$, et dont le fruit est transformé par les populations Bafulero en bière indigène fortement alcoolisée (10 à $15 \%$ ) s'appelant "pombé», qui n'est, par conséquent, pas facilement à obtenir par les Bafulero de la montagne. Ceux-ci se distinguent pour cela des Bafulero dégénérés de la plaine par leur grande taille, leur vitalité et leur intelligence.

Chez la population montagnarde j'ai pu observer une coutume très particulière qui concerne le placenta. Après la naissance, les femmes qui ont assisté à l'accouchement, procèdent à une sorte d'enterrement du placenta, en le couvrant légèrement de terre à côté d'un des petits chemins derrière la maison. Sur le placenta, elles déposent un pot dont la paroi est percée de deux petits trous - cela doit permettre à l'esprit du placenta de "voir ce qui se passe en dehors du pot».

Les femmes entourent ce pot de terre et de pierres (figure 1) et couvrent le petit tumulus, qui atteint une hauteur d'environ $60 \mathrm{~cm}$, d'une pierre plate en forme de dalle. Près de Mulenghe, à deux jours de marche de Sanghe, j'ai pu observer cinq de ces petits "tumuli»; en traversant d'autres villages des Bafulero de la montagne, j'en ai rencontré une autre dizaine avec des pots à deux trous, intacts, cassés ou en tessons - derniers 
vestiges de ces pots. Cette coutume est pratiquée pour protéger le nouveau-né contre les mauvais esprits de la montagne. C'est deux mois après la naissance qu'on se réunit à l'endroit où le placenta est enterré. Les parents, les membres de leurs familles et leurs amis, y célèbrent une fête qui dure plusieurs jours et nuits. On arrose le tumulus de bière indigène. Cette fête terminée, on ne s'occupe plus de lui et il sera lentement détruit par les pluies (figure 2).

La même coutume existe également chez les Bambutti y résidant, que les Bafulero appellent "Wambotte» et qu'ils emploient comme serviteurs et chasseurs. C'est un groupe de pygmées d'assez grande taille $(140$ à $155 \mathrm{~cm})$ et dont la forme typique du nez est le seul vestige physique de leur race.

Plus tard, on arrive dans la vallée de Lubuka. Celle-ci, bien abritée et très fertile, est parsemée de villages Bafulero. A chaque passage nous avons dû y rester plusieurs jours. L'affluence des indigènes ne voyant presque jamais de médecin, était grande. Des malades et des indigènes désireux de faire "trancher leurs palabres» (liquider leurs différends) attendaient leur tour.

On nous a montré un village dont presque tous les habitants âgés étaient atteints de grands goitres cystiques, en tant que les villages voisins n'en indiquaient aucun cas.

Près de ce village se trouve une grotte appelée Luala, dont les couches, de 4 à $6 \mathrm{~m}$ de profondeur, sont riches en ossements. Le manque de temps ne nous a pas permis d'y

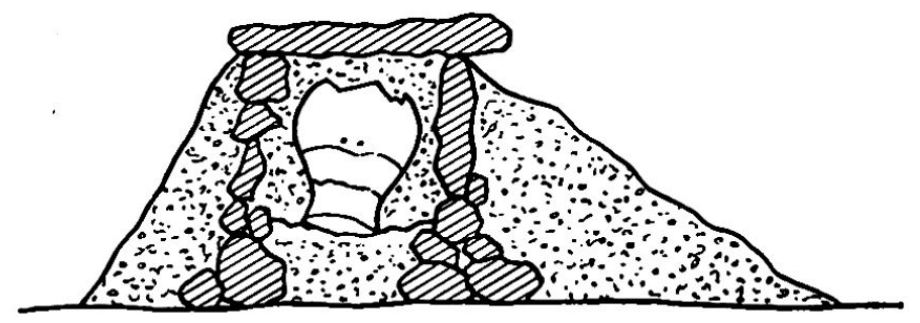

Fig. 1. Coupe d'un nouveau tumulus à placenta à Mulenge (sur la piste de Sanghe à Luemba). Dess. G. Leuzinger, Zurich

faire des fouilles systématiques. En descendant du village de Lubuka à l'Ulindi, on arrive à une source sulfureuse chaude, qui est très fréquentée par les indigènes malades.

Par la suite nos porteurs ont eu de grandes difficultés à traverser le col qui sépare la vallée de l'Ulindi des vallées des deux rivières Elila, parce que le point culminant de la piste atteint une altitude d'environ $3000 \mathrm{~m}$. Il s'appelle "Kilima mbua», c'est-à-dire "grande montagne». Nous avons pu y constater une autre coutume particulière: Les porteurs qui, pendant la montée avaient ramassé chacun une pierre, la déposaient en arrivant au col, sur un tas de pierres de 2 à $3 \mathrm{~m}$ de diamètre et d'une hauteur de 60 à $80 \mathrm{~cm}$ se trouvant à côté du chemin, tandis qu'ils prononçaient les mots «Kilima mbua, aidemoi». Quelques porteurs Bafulero de la plaine qui n'avaient jamais auparavant fait ce voyage, furent fort consternés en faisant sur ce col la connaissance de la neige et du gel; ils se plaignaient en disant: "C'est blanc comme la farine de manioc, mais cela mord les orteils comme les dents des serpents.»

Au sud-ouest du "Kilima mbua» il n'y a plus de villages. C'est une sorte de "No man's land» qui commence et qui s'étend à plusieurs journées de marche vers l'ouest, vers le Maniema. Le pays très accidenté de ces grandes altitudes est uniquement habité par quelques groupes de Banya-Ruanda et de Barundi, dont un certain nombre de métis Watussi, facilement reconnaissables à leur grande taille, leurs traits hamitiques et leur constitution asthénique. Ces bergers pratiquent la transhumance, ils viennent du RuandaUrundi et gardent leur bétail au pâturage en se nourrissant uniquement de lait caillé, de fromage et de viande de chasse. Ils vivent en petits groupes de 3 à 4 hommes dans des petites huttes de bambou, entourées d'une enceinte palissadée pour y protéger 
le bétail contre les nuits froides, les tempêtes et les léopards. Il leur arrive aussi de devoir chasser des buffles roux de montagne qui se mèlent à leurs troupeaux et dont ils ne désirent pas de progéniture pour leur bétail. Il est extrêmement pénible d'explorer les habitations, car en y pénétrant, on est envahi par des milliers de puces affamées qui montent en vitesse sur les jambes de ceux qui s'y aventurent.

Pendant trois jours notre safari continue, toujours sur les pistes du bétail. Ce pays montagneux est d'un grand charme et il nous réserve des surprises à chaque tournant du chemin: on passe par des monts et des vallées, par des forêts et des marais; on descend aux sources de l'Elila qu'on traverse à gué; on monte de nouveau à côté de chutes d'eau qui tombent des rochers, et on arrive dans une forêt de bambous habitée par des gorilles de montagne. Cette région n'est visité que de temps en temps par quelques chasseurs Babembe, proches parents des Warega du Maniema et très friands de viande de

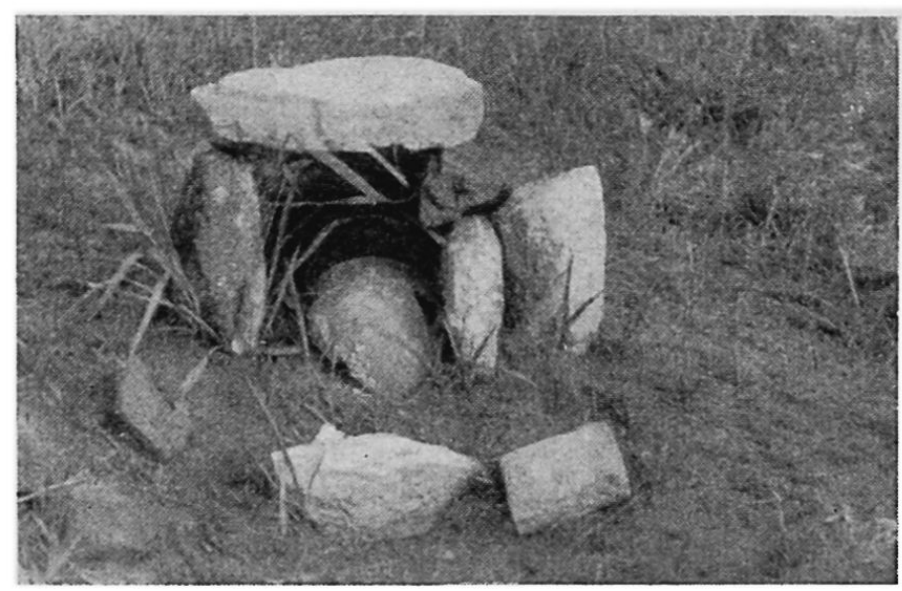

Fig. 2. Tumulus à placenta partiellement détruit par les pluies, situé derrière une maison de Mulenge. Photo:F. Hautmann

gorilles. Finalement on arrive dans le camp de mine de Luemba, construit en briques sèches avec des toits en tôle ondulée, situé sur une pente à environ $2000 \mathrm{~m}$ d'altitude. A l'exception de la viande, fournie par les bergers du Ruanda-Urundi, tout ce dont sa population d'une centaine de travailleurs avec leurs femmes et enfants a besoin, doit être amené à dos d'homme. Ainsi la caravane est attendue avec impatience et fêtée avec joie, non seulement par les noirs, mais surtout par le chef européen du camp de mine qui ne voit que deux ou trois autres Européens par an dans ce coin perdu des montagnes de l'Afrique centrale.

\section{ETHNOGRAPHISCHE STUDIEN IM «ITOMBWE» (KIVU-DISTRIKT, BELGISCH-KONGO)}

Das «Itombwe» ist ein im Nordwesten des Tanganyikasees gelegenes Bergmassiv. An seinen Hängen siedeln im Norden die Bafulero, die sich in Tal- und Berg-Bafulero unterscheiden. Bei den BergBafulero besteht die eigenartige Sitte, die Plazenta nach der Geburt unter einer tumulusförmigen Steinsetzung zu bestatten, womit das neugeborene Kind vor drohenden Gefahren geschützt werden soll. Das Weideland im Innern des Massivs wird von den Ban y - $\mathrm{R}$ u a nda und den Ba rundi aus dem RuandaUrundi mit ihren Rinderherden aufgesucht. Sie betreiben dort eine Art primitiver Alpwirtschaft.

\section{STUDII ETNOGRAFICI IN «ITOMBWE»(DISTRETTO DI KIVU, CONGO BELGO)}

«Itombwe» è un massivo di montagne situato nel nord-ovest del lago Tanganyika. Ai suoi pendii vivono nel nord i Bafulero, popolazione che si divide in abitanti di montagna e delle valli. I Bafulero di montagna praticano il costume di sepolcrare la placenta dopo la nascita sotto un tumulo di pietre nell'intenzione di proteggere il bambino neo-nato dai pericoli minaccianti. I pascoli all'interiore del massivo sono frequentati dai Banya-Ruanda e Barundi dal Ruanda-Urundi con le loro greggi di manzi. Praticono lì un genere di economia alpestre primitiva. 